\title{
Unravelling the Quantum Maze
}

\author{
María Esther Burgos \\ Department of Physics, University of Los Andes, Mérida, Venezuela \\ Email: mburgos25@gmail.com
}

How to cite this paper: Burgos, M.E. (2018) Unravelling the Quantum Maze. Journal of Modern Physics, 9, 1697-1711. https://doi.org/10.4236/jmp.2018.98106

Received: June 15, 2018

Accepted: July 28, 2018

Published: July 31, 2018

Copyright $\odot 2018$ by author and Scientific Research Publishing Inc. This work is licensed under the Creative Commons Attribution International License (CC BY 4.0).

http://creativecommons.org/licenses/by/4.0/

\begin{abstract}
The restoration of philosophical realism as the basis of quantum mechanics is the main aim of the present study. A spontaneous projection approach to quantum theory previously formulated achieved this goal in cases where the Hamiltonian does not depend explicitly on time. After discussing the most relevant flaws of orthodox quantum mechanics, a formulation of the spontaneous projections approach in the general case is introduced. This approach yields experimental predictions which in general coincide with those of the orthodox version and overcomes its main flaws.
\end{abstract}

\section{Keywords}

Quantum Weirdness, Quantum Measurements, Spontaneous Quantum Jumps

\section{Introduction}

The foundations of quantum mechanics were laid in the period 1900-1926. Some of its achievements were introduced and discussed at the Fifth Solvay Congress (1927). Even though the theory seemed bizarre, it was accepted by the majority of participants at this meeting ([1], pp. 109-121). In 1930 Paul Dirac published the first formulation of quantum mechanics [2]. Two years later John von Neumann published Mathematische Grundlagen der Quantenmechanik [3]. Quantum mechanics was born.

These first versions of the theory share two characteristics: 1) The state vector $|\psi\rangle$ (wave function $\psi$ ) describes the state of an individual system. 2) They involve two laws of change of the system's state: Spontaneous (natural) processes, governed by the Schrödinger equation; and measurement processes, ruled by the projection postulate. This postulate gives an account for projections (collapses, reductions or quantum jumps) caused by measurements. Many other versions of quantum theory followed. Those where $|\psi\rangle$ describes the state of an individual 
system and the projection postulate is included among its axioms are generally called standard, ordinary or orthodox quantum mechanics (OQM), sometimes referred to as the Copenhagen Interpretation.

From its inception OQM, and in particular its projection postulate, was the target of merciless criticism. Many scientists denounced what they considered its flaws. Among them, 1) it is incompatible with determinism; 2) it implies a kind of action-at-a-distance; and 3) it renounces philosophical realism. In addition, OQM presents a conflict with conservation laws which has been largely ignored [4] [5] [6] [7] [8] and carries the seeds of incoherence and contradictions [9] [10].

In 1931 Albert Einstein rightfully proclaimed: "the belief in an external world independent of the perceiving subject is the basis of all natural science" [11]. The restoration of philosophical realism as the basis of quantum mechanics is hence worth being pursued. The corresponding change of formalism should be realized, however, keeping as much as possible the experimental predictions of OQM, a theory imposingly successful [12].

This is the main aim of the spontaneous projection approach (SPA), a version of quantum theory previously formulated for cases where the Hamiltonian does not depend explicitly on time. It achieved this goal to a certain degree: it does not modify the Schrödinger equation and recovers a version of Born's postulate where no reference to measurements is made [13] [14] [15]. But the fact that it cannot account for cases where the Hamiltonian depends explicitly on time was a flaw which became increasingly apparent during our critical review of time dependent perturbation theory (TDPT) and forced us to conclude that $O Q M$ weirdness is not limited to the measurement problem [9] [10].

The version of SPA introduced in the present paper is more general than the previous one for it includes cases where the Hamiltonian depends explicitly on time. It keeps, however, the essential traits of SPA first version and yields, as far as we can see, the same experimental predictions obtained from OQM.

\section{Philosophical Realism, Quantum Measurements and Scientific Problems}

We uphold philosophical realism. We did in the first version of SPA and adopt the same epistemology as the basis of our present, more elaborated and general formulation of SPA. Our philosophical starting point can be stated as follows: 1) the things physics is about are supposed to exist, whether they are observed or not; 2) every scientific theory represents things through conceptual models; and 3) the adequacy of a theory (and corresponding models) to the things it refers to must take experimental results into account. In agreement with the philosophical point of view we adopt, "there are no definitive theories or models in (factual) science, because scientific knowledge is always of a hypothetical and never of a final nature" [16] [17]. More on this subject in ([18], p. 86).

According to Mario Bunge, "the main epistemological problem about quantum theory is whether it represents real (autonomously existing) things, and 
therefore whether it is compatible with epistemological realism. The latter is the family of epistemologies which assume that a) the world exists independently of the knowing subject, and b) the task of science is to produce maximally true conceptual models of reality..." ([19], pp. 191-192). He adds: "The main pillar of the non-realist interpretations of quantum theory is a certain view on measurement and on the projection (reduction) of the state function that is involved in measurement... [Sometimes] 'measurement' is misused to denote any interaction of an entity with the environment... However, the worst misconception of measurement is its identification with the subjective experience of taking cognizance of the outcome of measurement" ([19], pp. 192-193). For instance, in von Neumann's view, a complete measurement involves the consciousness of the observer ([1], pp. 481-482) ([20], pp. 418-421). "By assuming that observation escapes the laws of physics... the orthodox view treats measurement as an unphysical process...” ([19], p. 200).

In his answer to the question "what can be observed?" Bell quotes Einstein saying "it is theory which decides what is 'observable'. I think he was right-'observation' is a complicated and theory-laden business. Then that notion should not appear in the formulation of fundamental theory" ([21], p. 208; emphases added). Bell exposes to ridicule the supposedly necessary intervention of an observer to cause projections when he asks: "What exactly qualifies some physical system to play the role of 'measurer'? Was the wave function of the world waiting to jump for thousands of millions of years until a single-celled living creature appeared? Or did it have to wait a little longer, for some better qualified system... with a $\mathrm{PhD}$ ? If the theory is to apply to anything but highly idealized laboratory operations, are we not obliged to admit that more or less 'measurement-like' processes are going on all the time, more or less everywhere? Do we have jumping all the time?" ([21], p. 209).

Some authors dealing with the measurement problem avoid reference to the observer, but assume that measuring devices are macroscopic. Concerning this hypothesis Max Jammer highlights: "as long as a quantum mechanical one-body or many-body system does not interact with a macroscopic object, as long as its motion is described by the deterministic Schrödinger time-dependent equation, no events could be considered to take place in the system... If the whole physical universe were composed only of microphysical entities, as it should be according to the atomic theory, it would be a universe of evolving potentialities (timedependent $\psi$-functions) but not of real events" ([1], p. 474).

A few authors have considered the possibility that projections may happen at the microscopic level, that they are not necessarily the result of the interaction between a quantum system and a macroscopic object [22] [23]. We agree. Collapses are a kind of spontaneous processes occurring in nature. In order to take place, they require neither the intervention of observers nor the interaction of a microscopic (quantum) system with a macroscopic (classical) measuring device [13]. Reductions may also happen in tiny isolated systems. 
According to Bunge "the question of reality has nothing to do with scientific problems such as whether all properties have sharp values, and whether all behavior is causal" ([19], p. 192; emphases added). He adds: "unfortunately the two main controversies, those over realism and determinism (or hidden variables), have often been mixed up-and this by scientists of the stature of Einstein and de Broglie, Bohm and d'Espagnat. Yet the two issues are quite different: whereas the problem of realism is epistemological, that of hidden variables is ontological..." ([19], p. 168). We agree. But the list of scientific problems which have nothing to do with the question of reality ought to include at least three additional issues not mentioned by Bunge: the kind of action-at-a-distance pointed out by Einstein in the Fifth Solvay Congress ([1], p. 116); the validity of conservation laws [8]; and OQM incoherence and contradictions introduced through TDPT [9] [10]. Let us briefly consider these three issues.

\subsection{OQM Implies a Kind of Action-at-a-Distance}

The contradiction between the individual interpretation of the wave function $\psi$ and the postulate of relativity was first pointed out by Einstein in the Fifth Solvay Congress. In the case of a particle that, after diffraction in a slit arrives at a certain point of a scintillation-screen, he pointed out that the theory of quanta can be considered from two different viewpoints: I) The de Broglie-Schrödinger waves do not represent one individual particle but rather an ensemble of particles distributed in space. Accordingly, the theory provides information not on an individual process but rather on an ensemble of them... II) Quantum mechanics is considered a complete theory of individual processes. Hence, "each particle moving toward the screen is described as a wave packet which, after diffraction, arrives at a certain point $\mathrm{P}$ on the screen, and $|\psi(r)|^{2}$ expresses the probability (probability density) that at a given moment one and the same particle shows its presence at $r . . . "([1]$, pp. 115-116).

Einstein objected to the second possibility on the following grounds: "If $|\psi|^{2}$ is interpreted according to II, then, as long as no localization has been effected, the particle must be considered as potentially present with almost constant probability over the whole area of the screen; however, as soon as it is localized, a peculiar action-at-a-distance must be assumed to take place which prevents the continuously distributed wave in space from producing an effect at two places in the screen... 'It seems to me,' Einstein continued, 'that this difficulty cannot be overcome unless the description of the process in terms of the Schrödinger wave is supplemented by some detailed specification of the localization of the particle during its propagation... If one works only with Schrödinger waves, the [individual] interpretation of $|\psi|^{2}$, I think, contradicts the postulate of relativity'." ([1], p. 116; emphases added).

As early as 1927 (during the Fifth Solvay Congress) Einstein proved that the idea that quantum mechanics is a complete theory of individual processes renders inescapable the notion of instantaneous quantum jumps [15] [24]. His conclusion is the result neither of a sophisticated experiment nor of a cumbersome 
argument. It comes from logical reasoning applied to a very simple though experiment. To our knowledge, nobody has shown him wrong.

Eight years later, Einstein et al. published their celebrated article Can Quantum-Mechanical Description of Physical Reality Be Considered Complete? [25]. In this paper, best known as the EPR paradox, they referred to a system of two particles in an entangled state. In 1964 John Bell proved that no theory of nature that obeys local realism (and so satisfies certain inequalities) can reproduce all the predictions of quantum theory [26]. The contradiction between Bell's inequalities and quantum mechanics was submitted to experimental test by Stuart Freedman and John Clauser in 1972 [27]. Many other experiments followed this pioneer contribution. In general they yielded results in agreement with quantum mechanics. We have addressed the EPR paradox and related contributions in previous papers [15] [16] [24].

OQM implies what Einstein named "a spooky action-at-a-distance." There was a time when this notion was rejected by the majority of physicists. Nowadays it is accepted by almost everybody. This change of attitude can be retraced to the series of experiments aiming to test Bell's inequalities, in particular that performed by Hensen et al. in 2015 [28] and quantum teleportation obtained quite recently [29]. Let us add that, even though non-locality has been mostly associated to systems of particles in an entangled state, non-locality has been proven to also be present in experiments performed with individual particles. This can be easily verified with experimental techniques accessible to everybody [24].

The experiment performed by Hensen et al. has prompted Howard Wiseman to claim Death by experiment for local realism [30]. Local realism has died. Let us stress, however, that neither realism implies locality nor locality implies realism. These two concepts have been unduly mixed up. Non-locality really happens; the notion that every process is local lacks justification. This does not imply, however, renouncing realism.

\subsection{OQM Is at Variance with Determinism and Conservation Laws}

OQM conflicts determinism. To sample the reaction generated a century ago by such a conflict, let us recall that during the general debate of the Fifth Solvay Congress, its chairman Hendrick Lorentz objected the rejection of determinism, as proposed by the majority of speakers. He concluded with a desperate remark: "Je pourrais toujours garder ma foi déterministe pour les phénomènes fondamentaux... Est-ce qu'un esprit plus profond ne pourrait pas se rendre compte des mouvements de ces électrons? Ne pourrait-on pas garder le déterminisme en faisant l'objet d'une croyance? Faut-il nécessairement exiger l'indéterminisme en principe?" [I could always keep my faith in the determinism of fundamental phenomena... A more powerful mind could not give an account for the motion of these electrons? Determinism could be not kept as believe? Is it necessary to renounce determinism by principle?] ([1], p. 114). 
The relation between determinism and conservation laws was first pointed out by Henry Poincaré. Concerning the law of conservation of energy, he declared: “cette loi] ne peut avoir qu'une signification, c'est qu'il y a une propriété commune à tous les possibles; mais dans l'hypothèse déterministe il n'y a qu'un seul possible et alors la loi n'a plus de sens. Dans l'hypothèse indéterministe, au contraire, elle en prendrait un..." [this law cannot have more than one meaning, it is that there is a property shared by every possible; but in the determinist hypothesis there is a unique possible, then the law has no sense any more. In the indeterminist hypothesis, by contrast, it would have a sense...] ([31], p. 161).

This remark is pertinent: since OQM explicitly states that quantum measurements are processes not ruled by deterministic laws, one should suspect that conservation laws are not necessarily valid in such processes [15]. We have dealt with this subject for some time and concluded that, in the framework of OQM, conservation laws are strictly valid in spontaneous processes (ruled by a deterministic law); but have only a statistical sense in measurement processes (ruled by probability laws) [4] [5] [6] [7] [8]. Taking into account Poincaré's remark, this should not be surprising: in the first case conservation laws are theorems which can be derived from an axiom which is not valid in the second case.

\subsection{OQM Is Incoherent and Contradictory}

OQM marvelous success in the area of experimental predictions requires, in general, the application of TDPT. It is agreed that the method provided by TDPT must be used in all problems involving a consideration of time, including spontaneous time dependent processes; see for instance ([2], p. 168). This is the case of absorption and emission of light and of processes occurring in semiconductors. To give an account for such spontaneous processes, however, TDPT requires the application of a law which is not valid in spontaneous processes. This is a flagrant incoherence we have not noticed in the literature [9].

Let us sketch our argument: Consider a system with Hamiltonian $\varepsilon$ which does not depend explicitly on time. It will be called the unperturbed Hamiltonian of the system. Its eigenvalue equations are

$$
\varepsilon\left|\phi_{n}\right\rangle=E_{n}\left|\phi_{n}\right\rangle
$$

where $E_{n}(n=1,2, \cdots)$ are the eigenvalues of $\varepsilon$ and $\left|\phi_{n}\right\rangle$ the corresponding eigenstates. For simplicity we assume $\varepsilon$ spectrum to be entirely discrete and non-degenerate; all the states referred to in this study are normalized.

Let us suppose that at initial time $t=0$ the system is in the stationary state $\left|\phi_{j}\right\rangle$. A system in a stationary state will remain in that state forever: if for $t \geq 0$ the Hamiltonian were $\varepsilon$, the state vector at time $t$ would be

$$
|\psi(t)\rangle=\mathrm{e}^{-i E_{j} t / \hbar}|\psi(0)\rangle=\mathrm{e}^{-i E_{j} t / \hbar}\left|\phi_{j}\right\rangle
$$

Nevertheless, TDPT establishes that by applying a time dependent perturbation, transitions between different eigenstates of $\varepsilon$ can be induced and determines the probability corresponding to every particular transition ([2], pp. 
172-173).

If at $t=0$ a time dependent perturbation $\mathscr{W}(t)$ is applied, for $t \geq 0$ the total, perturbed Hamiltonian will be

$$
\mathscr{H}(t)=\varepsilon+\mathscr{W}(t)
$$

The perturbation $\mathscr{\omega}(t)$ causes the state $|\psi(0)\rangle$ to change. According to TDPT, the Schrödinger evolution leads the initial state $|\psi(0)\rangle=\left|\phi_{j}\right\rangle$ to the state

$$
|\psi(t)\rangle=\mathcal{U}(t, 0)|\psi(0)\rangle=\mathcal{U}(t, 0)\left|\phi_{j}\right\rangle
$$

where $\mathcal{U}(t, 0)$ is, by definition, the evolution operator corresponding to the Hamiltonian $\mathscr{H}(t)$. The probability of a transition taking place from state $\left|\phi_{j}\right\rangle$ to state $\left|\phi_{k}\right\rangle$ during the time interval $(0, t)$ is

$$
\mathcal{P}_{0, t}\left(E_{j} E_{k}\right)=\left|\left\langle\phi_{k}|\mathcal{U}(t, 0)| \phi_{j}\right\rangle\right|^{2}
$$

TDPT deals with processes having two clearly different stages. In the first-during the time interval $(0, t)$-a Schrödinger evolution leads the system's state from $|\psi(0)\rangle$ to $|\psi(t)\rangle$ given by Equation (4) with certitude; this change is automatic. In the second an instantaneous projection of $|\psi(t)\rangle$ to a stationary state $\left|\phi_{k}\right\rangle$ is ruled by probability laws [9]. According to OQM, the Schrödinger equation governs every spontaneous process; Born's postulate and/or the projection postulate apply only when measurements are performed, resulting in a quantum jump. "The fact that TDPT requires the application of postulates concerning measurements to give an account for processes supposedly spontaneous (v.g. absorption and emission of light) is at the very heart of OQM incoherence”[9].

A further critical review of TDPT unveiled a contradiction reminiscent of Zeno's paradoxes concerning motion [10]. The argument can be sketched as follows.

Referring to a system in the initial state $|\psi(0)\rangle=\left|\phi_{j}\right\rangle$, Dirac asserts: "at time $t$ the ket corresponding to the state in Schrodinger's picture will be $|\psi(t)\rangle=\mathcal{U}(\mathrm{t}, 0)\left|\phi_{j}\right\rangle$ according to Equation (4). The probability of the $E_{n}$ 's then having the values $E_{k}$ is $\mathscr{P}_{0, t}\left(E_{j} E_{k}\right)$ given by Equation (5). For $k \neq j$, $\mathscr{P}_{0, t}\left(E_{j} E_{k}\right)$ is the probability of a transition taking place from state $\left|\phi_{j}\right\rangle$ to state $\left|\phi_{k}\right\rangle$ during the time interval $(0, t)$, while $\mathscr{P}_{0, t}\left(E_{j} E_{j}\right)$ is the probability of no transition taking place at all. The sum of $\mathscr{P}_{0, t}\left(E_{j} E_{k}\right)$ for all $k$ is, of course, unity" ([2], p. 172-173; emphases added).

The transition taking place from state $\left|\phi_{j}\right\rangle$ to state $\left|\phi_{k}\right\rangle$ during the interval $(0, t)$ involves an instantaneous jump, i.e. a discontinuous change at time $t$. Since the sum of probabilities corresponding to all possible discontinuous changes at time $t$ is unity, no room is left for a non-null probability corresponding to a process continuous at this instant [10]. Dirac does not impose any particular condition on the instant $t$. Hence the process cannot be continuous at any instant, the state vector at time $t$ cannot be $|\psi(t)\rangle=\mathscr{U}(\mathrm{t}, 0)\left|\phi_{j}\right\rangle$ and transitions between stationary states during the time interval $(0, t)$ as referred to in TDPT 
cannot take place; the system remains stuck to its initial stationary state. "Paraphrasing Zeno, these kinds of transitions between stationary states are nothing but illusions" [10].

Except Albert Messiah, no other author known to us imposes any particular condition on the interval $(0, t)$. By contrast, Messiah explicitly assumes that an instantaneous measurement is performed at time $t$ ([32], p. 621). In absence of measurement, the Schrodinger evolution follows and the probability of a transition taking place from $\left|\phi_{j}\right\rangle$ to $\left|\phi_{k}\right\rangle$ during the interval $(0, t)$ is null. To avoid the "quantum Zeno contradiction" Messiah pays the price of assuming that an instantaneous measurement is performed every time a transition between two stationary states takes place [10].

Quantum weirdness has been traditionally associated with the measurement problem. To solve it, different authors have suggested several strategies. Among them are statistical interpretation of quantum mechanics [33], many worlds interpretation [34], decoherence [12] and continuous spontaneous localization theory [22]. We have addressed these and other proposed solutions to the measurement problem in previous papers [13] [14] [15]. Despite their value, these contributions do not solve the measurement problem, let alone OQM incoherence and the quantum Zeno contradiction just mentioned.

OQM weirdness is certainly not limited to the measurement problem. It is much more serious and justifies a radical revision of the theory [9] [10]. An overview of such a task follows.

\section{The Spontaneous Projection Approach}

Two kinds of processes irreducible to one another occur in nature: the strictly continuous and causal ones, which are governed by a deterministic law and those implying discontinuities, which are ruled by probability laws. This is the main hypothesis of SPA [13] [14] [15].

We explicitly discard the observer intervention and the interaction between the quantum system with a macroscopic measuring device as a source of projections. So the question is: what could then induce quantum jumps? SPA answers: the tendency the system's state has to jump to the eigenstates of operators representing conserved quantities. Let us establish this hypothesis in a formal way.

Let $\alpha$ be the self-adjoint operator representing the physical quantity $\alpha$ referred to the physical system $\zeta$. We assume that the Hamiltonian, denoted by $\varepsilon$, does not depend explicitly on time $t$. Then, if the operator $\alpha$ fulfills the conditions

$$
\frac{\partial \alpha}{\partial t}=0
$$

and

$$
[\alpha, \varepsilon]=0
$$


the system's state $|\psi(t)\rangle$ has the tendency to jump to the eigenstates of $\alpha$. We have shown, however, that this tendency is seldom realized [13] [14] [15].

Let us highlight the difference between this hypothesis and that adopted in continuous spontaneous localization theory. In the last approach collapses localize the wave function [22]. As a result, steady states cannot be attained [35]. By contrast, according to SPA in most cases projections lead the system to stationary states [13].

\subsection{The Statistical Sense of Conservation Laws}

We have previously asserted that the conflict of OQM with conservation laws has been largely ignored [4] [5] [6] [7] [8]. Let us briefly review this issue.

The mean value of the physical quantity $\alpha$ is by definition

$$
\langle\alpha\rangle(t)=\langle\psi(t)|\alpha| \psi(t)\rangle
$$

In Schrödinger evolutions the validity of Equation (6) and (7) ensures that $\langle\alpha\rangle(t)$ remains a constant in time for every state $|\psi(t)\rangle$ of $\zeta$. It is said that $\alpha$ is a constant of the motion and that $\alpha$ is conserved. By contrast, in processes ruled by another, different law from Schrödinger equation, the validity of Equations (6) and (7) does not guarantee that $\langle\alpha\rangle(t)$ remains a constant in time: if the process starts at $t_{0}$ and ends at $t_{f}$, it can result $\langle\alpha\rangle\left(t_{f}\right) \neq\langle\alpha\rangle\left(t_{0}\right)$ [8]. Hence the assertions " $\alpha$ is a constant of the motion" and " $\alpha$ is conserved" are not justified. However, the average of the changes $\delta\langle\alpha\rangle=\langle\alpha\rangle\left(t_{f}\right)-\langle\alpha\rangle\left(t_{0}\right)$ obtained by repeating the process many times, converges to zero [8].

Let us consider a set of $N$ orthonormal vectors: $\left|u_{1}\right\rangle,\left|u_{2}\right\rangle, \cdots\left|u_{N}\right\rangle\left(\left\{N_{u}\right\}\right.$ for short) such that the system's state at time $t$ can be written

$$
|\psi(t)\rangle=\sum_{j} c_{j}(t)\left|u_{j}\right\rangle
$$

where $c_{j}(t)=\left\langle u_{j} \mid \psi(t)\right\rangle$ and $j=1,2, \cdots, N$. The mean value of $\alpha$ at time $t$ is $\langle\alpha\rangle(t)$ given by Equation (8); in particular, if $|\psi(t)\rangle=\left|u_{j}\right\rangle$ this mean value is $\left\langle u_{j}|\alpha| u_{j}\right\rangle$. Then,

Postulate I: If Equations (6) and (7) are satisfied, the validity of

$$
\langle\psi(t)|\alpha| \psi(t)\rangle=\sum_{j}\left|c_{j}(t)\right|^{2}\left\langle u_{j}|\alpha| u_{j}\right\rangle
$$

is a necessary condition for the state $|\psi(t)\rangle$ given by Equation (9) may collapse to the vectors of the set $\left\{N_{u}\right\}$, i.e. for jumps like $|\psi(t)\rangle \rightarrow\left|u_{1}\right\rangle$, or $|\psi(t)\rangle \rightarrow\left|u_{2}\right\rangle, \cdots$ or $|\psi(t)\rangle \rightarrow\left|u_{N}\right\rangle$, may occur [13] [14] [15].

Postulate I recovers Poincarés assertion: In the indeterminist hypothesis, conservation laws have a statistical sense [13] [14] [15].

\subsection{The Concept of Preferential Set}

If there is a unique set of $N \geq 2$ orthonormal vectors: $\left|\varphi_{1}\right\rangle,\left|\varphi_{2}\right\rangle, \cdots,\left|\varphi_{N}\right\rangle$ ( $\left\{N_{\varphi}\right\}$ for short) such that 1 ) the state of the physical system $\zeta$ at time $t$ can be 
written

$$
|\psi(t)\rangle=\sum_{j} \gamma_{j}(t)\left|\varphi_{j}\right\rangle
$$

where 2) $\gamma_{j}(t)=\left\langle\varphi_{j} \mid \psi(t)\right\rangle \neq 0$ for every $j=1,2, \cdots, N$; 3) at least $(N-1)$ vectors belonging to the set $\left\{N_{\varphi}\right\}$ are eigenstates of the Hamiltonian $\varepsilon$ (i.e. stationary states); and 4) every self-adjoint operator $\alpha$ for which Equations (6) and (7) are valid satisfies the relation

$$
\langle\psi(t)|\alpha| \psi(t)\rangle=\sum_{j}\left|\gamma_{j}(t)\right|^{2}\left\langle\varphi_{j}|\alpha| \varphi_{j}\right\rangle
$$

we shall say that $\left\{N_{\varphi}\right\}$ is the preferential set of $\zeta$ in the state $|\psi(t)\rangle$ and the members of $\left\{N_{\varphi}\right\}$ will be called its preferential states.

Comment 1: According to this definition, a system $\zeta$ in the state $|\psi(t)\rangle$ can either have a unique preferential set including at least two preferential states or not have a preferential set at all.

Comment 2: The concept of the preferential set of $\zeta$ in the state $|\psi(t)\rangle$ adopted here coincides with that introduced in [10] and is different from our original concept of a preferential set of $\zeta$ in the state $|\psi(t)\rangle$ [13] [14]; the difference being that in the original definition the set $\left\{N_{\varphi}\right\}$ was not supposed to be unique, and condition (2) was not assumed to be valid.

Comment 3: Besides the concept of a preferential set of $\zeta$ in the state $|\psi(t)\rangle$, in previous papers we introduced the concepts of preferential basis and of maximal preferential set [13] [14]. Taking into account the present definition of the preferential set of $\zeta$ in the state $|\psi(t)\rangle$, the concepts of preferential basis and of maximal preferential set become superfluous. Hence they will not be referred to in the following.

We have so far assumed that the system's Hamiltonian $\varepsilon$ does not depend explicitly on time. Let us now consider cases where the system's Hamiltonian depends explicitly on time. It can be written

$$
\mathscr{H}(t)=\varepsilon+\mathscr{W}(t)
$$

where $\mathscr{\omega}(t)$ includes every term of the Hamiltonian which depends explicitly on time. Then we state

Postulate II: The preferential set (and its preferential states) of $\zeta$ in the state $|\psi(t)\rangle$ does not depend on the term $\%(t)$.

Examples of the determination of preferential states have been given elsewhere [10] [13] [14] [15].

\subsection{The Formalism of SPA}

SPA includes the primitive (undefined) notions: system, state, physical quantity (or dynamical variable) and probability. Note that except the last one, these primitive concepts coincide with those adopted in Jammer's axiomatic presentation of the formalism of quantum mechanics due to von Neumann ([1], p. 5).

Postulate A: To every system $\zeta$ corresponds a Hilbert space $\delta$ whose vectors 
(state vectors, wave functions) $|\psi(t)\rangle$ completely describe the states of the system.

Postulate B: To every physical quantity $\alpha$ corresponds uniquely a self-adjoint operator $\alpha$ acting in $\delta$. It has associated the eigenvalue equations

$$
\alpha\left|a_{k}^{v}\right\rangle=a_{k}\left|a_{k}^{v}\right\rangle
$$

( $v$ is introduced in order to distinguish between the different eigenvectors that may correspond to one eigenvalue $a_{k}$ ), and the closure relation

$$
\sum_{k, v}\left|a_{k}^{v}\right\rangle\left\langle a_{k}^{v}\right|=\mathfrak{g}
$$

is fulfilled (here $g$ is the identity operator). If $k$ or $v$ iscontinuous, the respective sum has to be replaced by an integral.

Comment I: The correspondence postulates A and B associate the primitive notions system, physical quantity and state of the system with mathematical entities. The same is true of von Neumann's quantum mechanics version reported in ([1], p. 5).

Postulate C: Continuous processes are governed by the Schrödinger equation

$$
i \hbar \frac{\mathrm{d}}{\mathrm{d} t}|\psi(t)\rangle=\mathscr{H}(t)|\psi(t)\rangle
$$

where $\mathscr{H}(t)$ is the Hamiltonian of the system, $\hbar$ Planck's constant divided by $2 \pi$ and $i$ the imaginary unity.

Comment II: The Schrödinger equation is a deterministic law. The solution $|\psi(t)\rangle$ of Equation (16) which corresponds to the initial condition $|\psi(0)\rangle$ is unique. The system's state evolves in correspondence with the equation

$$
|\psi(t)\rangle=\mathscr{X}(t, 0)|\psi(0)\rangle
$$

where $\mathcal{U}(t, 0)$ is the evolution operator corresponding to the Hamiltonian $\mathscr{H}(t)$; more details in ([2], p. 109) ([36], p. 137) ([37], p. 308) ([38], p. 41).

Postulate D: A discontinuous change of the system's state occurs if and only if $|\psi(t)\rangle$ jumps to one of its preferential states. If the system $\zeta$ in the state $|\psi(t)\rangle$ does not have preferential states, the process is necessarily continuous and governed by the Schrödinger equation.

Let us assume that the system $\zeta$ in the state $|\psi(t)\rangle$ has the preferential set $\left\{N_{\varphi}\right\}$. So we can write

$$
|\psi(t)\rangle=\sum_{k} \gamma_{k}(t)\left|\varphi_{k}\right\rangle
$$

where $k=1,2, \cdots, N$. Under these conditions we state

Postulate E: In the small time interval $(t, t+\mathrm{d} t)$ the state $|\psi(t)\rangle$ can undergo the following changes

$$
|\psi(t)\rangle \rightarrow|\psi(t+\mathrm{d} t)\rangle=\left|\varphi_{k}\right\rangle
$$

with probability 


$$
\mathrm{d} \mathscr{P}_{k}(t)=\left|\gamma_{k}(t)\right|^{2} \frac{\mathrm{d} t}{\tau(t)}
$$

or

$$
|\psi(t)\rangle \rightarrow\left|\psi_{\mathcal{\varkappa}}(t+\mathrm{d} t)\right\rangle=\mathcal{U}(t+\mathrm{d} t, t)|\psi(t)\rangle
$$

with probability

$$
\mathrm{d} \mathscr{P}_{\mathfrak{U}}(t)=1-\frac{\mathrm{d} t}{\tau(t)}
$$

Here

$$
\tau(t) \Delta \varepsilon(t)=\frac{\hbar}{2}
$$

and

$$
[\Delta \varepsilon(t)]^{2}=\left\langle\psi(t)\left|\varepsilon^{2}\right| \psi(t)\right\rangle-[\langle\psi(t)|\varepsilon| \psi(t)\rangle]^{2}
$$

Comment III: Since $|\psi(t)\rangle$ is normalized, during a small time interval $(t, t+\mathrm{d} t)$ the system in the state $|\psi(t)\rangle$ has a probability $\frac{\mathrm{d} t}{\tau(t)}$ to jump to one of its $N$ preferential states. If $\mathrm{d} t \ll \tau(t)$, the dominant process is the Schrödinger evolution [13].

Comment $I V$ : In general the parameter $\tau$ defined by Equation (23) depends on time $t$. But if $\tau$ is a constant, the state $|\psi(t)\rangle$ may be considered as an unstable state that can decay to one of its $N$ preferential states [13] [14] [15]. Let $\mathscr{P}_{\mathfrak{U}}(t)$ be the probability that the system's state has not jumped to any preferential state in the interval $(0, t)$. The well-known exponential decay law is then obtained:

$$
\mathscr{P}_{\mathscr{U}}(t)=\mathrm{e}^{-t / \tau}
$$

\section{Concluding Remarks}

Let us conclude with the following remarks.

On the one hand SPA and OQM share several traits:

1) Both theories refer to individual systems, not to ensembles of systems similarly prepared.

2) SPA does not modify OQM in a substantial way: It keeps without changes the Schrödinger equation and recovers a version of Born's postulate where no reference to measurement is made. So, in general its experimental predictions coincide with those of OQM [13] [14] [15].

3) Both theories imply a "spooky action-at-a-distance" which is a kind of action-at-a-distance easily verifiable with techniques accessible to everybody [24]. Since this effect actually happens, there is no reason to discard theories which imply it.

4) In SPA as in OQM conservation laws fail in individual processes involving 
quantum jumps.

On the other hand, SPA and OQM exhibit remarkable differences:

1) Unlike OQM, SPA is compatible with philosophical realism. In SPA there is no room for observers placed above the laws of nature.

2) The conspicuous notions of measurement and observation in OQM are alien to SPA. Differing from OQM, SPA fulfills Bell's requirement: "Tthe notion of observation] should not appear in the formulation of fundamental theory" ([21], p. 208; emphases added).

3) In OQM spontaneous processes are necessarily continuous and ruled by the Schrödinger equation, a deterministic law which yields automatic changes. By contrast, in SPA spontaneous processes are not necessarily continuous and ruled by the Schrödinger equation. If the system in the state $|\psi(t)\rangle$ has the preferential set $\left\{\left|\varphi_{1}\right\rangle,\left|\varphi_{2}\right\rangle, \cdots,\left|\varphi_{N}\right\rangle\right\}$, it can either follow a Schrödinger evolution or instantaneously jump to one of its preferential states.

4) In OQM reductions are ad-hoc, in SPA they are not surreptitious but explicitly included in the formalism.

5) OQM is incoherent and exhibits a contradiction reminiscent of Zeno's paradoxes of motion. SPA escapes these issues thanks to the hypothesis that collapses are natural processes [10].

In sum: while yielding experimental predictions which in general coincide with those of OQM, SPA enjoys a coherence which is absent from OQM and overcomes its main flaws.

\section{Acknowledgements}

We are indebted to Professor J. C. Centeno for many fruitful discussions. We thank Carlos Valero for his assistance with the transcription of the manuscript.

\section{Conflicts of Interest}

The authors declare no conflicts of interest regarding the publication of this paper.

\section{References}

[1] Jammer, M. (1974) The Philosophy of Quantum Mechanics. John Wiley \& Sons, New York.

[2] Dirac, P.A.M. (1958) The Principles of Quantum Mechanics. Clarendon Press Oxford, Oxford.

[3] von Neumann, J. (1932) Mathematische Grundlagen der Quantenmechanik. Springer, Berlin.

[4] Burgos, M.E. (1994) Physics Essays, 7, 69-71. https://doi.org/10.4006/1.3029115

[5] Burgos, M.E. (1997) Speculations in Science and Technology, 20, 183-187.

[6] Burgos, M.E., Criscuolo, F.G. and Etter, T. (1999) Speculations in Science and Technology, 21, 227-233. https://doi.org/10.1023/A:1005552504638

[7] Criscuolo, F.G. and Burgos, M.E. (2000) Physics Essays, 13, 80-84. https://doi.org/10.4006/1.3025430 
[8] Burgos, M.E. (2010) JMP, 1, 137-142. https://doi.org/10.4236/jmp.2010.12019

[9] Burgos, M.E. (2016) JMP, 7, 1449-1454. https://doi.org/10.4236/jmp.2016.712132

[10] Burgos, M.E. (2017) JMP, 8, 1382-1397. https://doi.org/10.4236/jmp.2017.88087

[11] Einstein, A. (1931) James Clerk Maxwell: A Commemoration Volume. Cambridge University Press, Cambridge.

[12] Tegmar, M. and Wheeler, J. (2001) Scientific American, 284, 68-75. https://doi.org/10.1038/scientificamerican0201-68

[13] Burgos, M.E. (1998) Foundations of Physics, 28, 1323-1346. https://doi.org/10.1023/A:1018826910348

[14] Burgos, M.E. (2008) Foundations of Physics, 38, 883-907. https://doi.org/10.1007/s10701-008-9213-5

[15] Burgos, M.E. (2015) The Measurement Problem in Quantum Mechanics Revisited. In: Pahlavani, M., Ed., Selected Topics in Applications of Quantum Mechanics, INTECH, Croatia, 137-173. http://dx.doi.org/10.5772/59209

[16] Burgos, M.E. (1983) Kinam, 5, 277-284.

[17] Burgos, M.E. (1987) Foundations of Physics, 17, 809-812. https://doi.org/10.1007/BF00733269

[18] Bunge, M. (1973) Philosophy of Physics. Reidel Publishing Company, Dordrecht, Boston, Lancaster.

[19] Bunge, M. (1985) Treatise on Basic Philosophy, Vol. 7, Philosophy of Science \& Technology. D. Reidel Publishing Company, Dordrecht, Boston, Lancaster.

[20] von Neumann, J. (1955) Mathematical Foundations of Quantum Mechanics. Princeton University Press, Princeton.

[21] Bell, M., Gottfried, K. and Veltman, M. (2001) John S. Bell on the Foundations of Quantum Mechanics. Word Scientific, Singapore.

[22] Ghirardi, G.C., Rimini, A. and Weber, T. (1986) Physical Review D, 34, 470-490. https://doi.org/10.1103/PhysRevD.34.470

[23] Primas H. (1990) The Measuremnet Process in the Individual Interpretation of Quantum Mechanics. In: Cini, M. and Lévy-Leblond, J.M., Eds., Quantum Theory Without Reduction, Adam Hilger, Bristol, 49-68.

[24] Burgos, M.E. (2015) JMP, 6, 1663-1670. https://doi.org/10.4236/jmp.2015.611168

[25] Einstein, A., Podolsky, B. and Rosen, N. (1935) Physical Review, 47, 777-780. https://doi.org/10.1103/PhysRev.47.777

[26] Bell, J.S. (1964) Physics, 1, 195-200. https://doi.org/10.1103/PhysicsPhysiqueFizika.1.195

[27] Freedman, S.J. and Clauser, J.F. (1972) Physical Review Letters, 28, 938-941. https://doi.org/10.1103/PhysRevLett.28.938

[28] Hensen, B., Bernien, H. and Dréau, A.E. (2015) Nature, 526, 682-686. https://doi.org/10.1038/nature15759

[29] Wikipedia. The Free Encyclopedia: Quantum Teleportation. https://en.wikipedia.org/wiki/Quantum_teleportation

[30] Wiseman, H. (2015) Nature, 526, 649-650. https://doi.org/10.1038/nature15631

[31] Poincaré, H. (1906) La science et l'hypothèse. Flammarion, Paris.

[32] Messiah, A. (1965) Mécanique Quantique. Dunod, Paris.

[33] Ballentine, L.E. (1970) Reviews of Modern Physics, 42, 358-381. 
https://doi.org/10.1103/RevModPhys.42.358

[34] Wikipedia. The Free Encyclopedia: Many-Worlds Interpretation. https://en.wikipedia.org/wiki/Many-worlds_interpretation

[35] Ballentine, L.E. (1991) Physical Review A, 43, 9-12. https://doi.org/10.1103/PhysRevA.43.9

[36] Bes, D.R. (2004) Quantum Mechanics. Springer, Berlin. https://doi.org/10.1007/978-3-662-05384-3

[37] Cohen-Tannoudji, C., Diu, B. and Laloë, F. (1977) Quantum Mechanics. John Wiley \& Sons, New York, London, Sydney, Toronto.

[38] Yndurain Muñoz, F.J. (2003) Mecánica Cuántica. Editorial Ariel S.A., Barcelona. 